\title{
Validação da dose planar e dose pontual segundo o protocolo TG-119 da AAPM para a técnica VMAT no acelerador linear Halcyon Validation of the planar dose and point dose according to the AAPM TG-119 protocol for VMAT technique on Halcyon linear accelerator
} Diego S. Teixeira1, Daniela R. Estácio ${ }^{1}$, Patrícia Sbaraini ${ }^{1}$, Ana M. Marques da Silva ${ }^{2}$

${ }^{1}$ Serviço de Radioterapia, Hospital São Lucas, PUCRS, Porto Alegre, Brasil ${ }^{2}$ Laboratório de Computação em Imagens Médicas Escola Politécnica, PUCRS, Porto Alegre, Brasil

\begin{abstract}
Resumo
Este trabalho tem por objetivo apresentar a validação da técnica de radioterapia em arco modulada volumetricamente (VMAT) no equipamento Halcyon, da Varian®, utilizando o protocolo TG-119 da American Association of Physicists in Medicine (AAPM). O protocolo TG-119 reúne diversas estruturas alvos para planejamento, bem como os objetivos definidos para otimização. O planejamento inverso foi realizado no software Eclipse, cujos planos foram executados no acelerador linear Halcyon, com uma energia máxima de 6MeV-FFF (Flattening Filter Free) para fótons. Os resultados obtidos mostraram que a média das medidas de dose pontual foi de $0,4 \%$ de diferença, e para análise gamma, a concordância foi de $98,72 \%$ dos pontos, passando no critério. Os limites de confiança alcançados para região de alta dose, região de baixa dose e análise planar de dose ficaram dentro dos valores estipulados no protocolo AAPM TG-119. Assim, conclui-se que o sistema de planejamento utilizado com a técnica VMAT está adequado para utilização diária no Halcyon, uma vez que os valores encontrados estão dentro dos limites estipulados pelo protocolo TG-119 da AAPM.

Palavras-chave: radioterapia, VMAT, protocolos, estudos de validação.
\end{abstract}

\begin{abstract}
This study aims to present the validation of the volumetrically modulated arc radiotherapy technique (VMAT) in the Halcyon equipment, by Varian $\AA$, using the TG-119 protocol of the American Association of Physicists in Medicine (AAPM). The TG-119 protocol brings together several target structures for planning as well as the objectives defined for optimization. The reverse planning was carried out in the Eclipse software. The plans were executed in the linear accelerator Halcyon with a maximum energy of 6MeV-FFF (Flattening Filter Free) for photons. The results obtained showed that the difference between the planned dose and the average of the point dose measurements was $0.4 \%$ and for gamma analysis, the agreement was in $98.72 \%$ of the points, thus passing the gamma criterion. The confidence limits reached for the high dose region, low dose region and planar dose analysis were within the values stipulated in the AAPM TG-119 protocol. Thus, it was concluded that the planning system used with the VMAT technique is suitable for daily use in Halcyon, since the values found are within the limits stipulated by the AAPM protocol TG-119.

Keywords:radiotherapy, VMAT, protocols, validation studies.
\end{abstract}

\section{Introdução}

As técnicas de entrega de dose ao paciente vêm sendo cada vez mais aprimoradas com a implementação de novas tecnologias, visando a aumentar a eficiência do tratamento e diminuir a toxicidade aos órgãos adjacentes ao volume de tratamento, causada pela radiação ionizante de alta energia.

Uma das mais recentes técnicas de radioterapia é a radioterapia em arco modulada volumetricamente (VMAT), que utiliza a modulação da intensidade do feixe de radiação de maneira volumétrica para atingir o alvo e poupar ao máximo as estruturas adjacentes. Essa técnica promete resultados melhores ou semelhantes em relação às técnicas de radioterapia de intensidade modulada e de radioterapia conformacional tridimensional (1).

O conceito de terapia com arco modulado volumétrico (VMAT, do inglês Volumetric Modulated Arc Therapy) foi proposto pela primeira vez em 1995, cujo trabalho desenvolvido por Otto (2) levou ao desenvolvimento de uma abordagem comercial de VMAT chamada RapidArc (Varian Medical Systems,Palo Alto,CA). Essa técnica oferece mais graus de liberdade para otimização e uma melhor entrega da dose.

Antes da implementação de uma técnica como a do VMAT para ser utilizada na rotina clínica de um serviço de radioterapia, deve-se comissionar o equipamento que utilizará tal técnica a fim de verificar a sua eficácia em um sistema de planejamento como o Eclipse, por meio de estruturas alvo superpostas em imagens de tomografia computadorizada de um objeto simulador. Deve-se executar o tratamento planejado e medir a dose, comparando-a com a dose calculada (3).

O protocolo TG-119, que foi criado pela American Association of Physicists in Medicine (AAPM) (4), é um documento de referência que nos fornece orientações de como devemos proceder com os planejamentos propostos, com os testes, bem como com os limites aceitáveis a partir da comparação dosimétrica dos resultados. O protocolo apresenta valores que nos fornecem a referência para limites de confiança esperados no pós-comissionamento, fornecendo os objetivos de dose planar no alvo desejado e em estruturas críticas adjacentes ao alvo 
para diferentes conjuntos de testes, tais como, multialvo, simulações de próstata, cabeça e pescoço e um alvo em forma de $\mathrm{C}$ com duas restrições (constraints) diferentes (fácil e difícil).

O Halcyon é um acelerador linear inovador na radioterapia em nível mundial, tendo em vista que possui um sistema de colimação de lâminas mais rápido, alta taxa de dose $(800 \mathrm{UM} / \mathrm{min})$ repercutindo no tempo em que a dose é entregue ao alvo, bem como alto giro do gantry, atingindo valores 4 vezes maiores do que o gantry de um acelerador linear típico. Essas características motivaram a realização desse trabalho cujo objetivo é a validação da técnica de radioterapia em arco modulada volumetricamente (VMAT) no equipamento Halcyon 2.0, da Varian $\AA$, em conformidade com os testes propostos pelo TG119 da American Association of Physicists in Medicine AAPM (4).

\section{Materiais e Métodos}

Para a realização das medidas de dose, conforme orientações contidas no protocolo TG-119 da AAPM (4), foram realizadas algumas adaptações, utilizando um phantom de $15 \mathrm{~cm}$ de espessura, com placas de $12 \mathrm{~cm}$ de água sólida, além de $3 \mathrm{~cm}$ de placas de acrílico, conforme é mostrado na Figura 1.

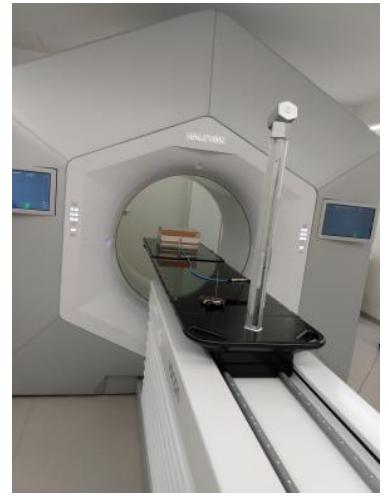

Figura 1. Phantom de placas de água sólida e acrílico junto com a câmara de ionização.

As medidas foram realizadas para avaliar a diferença na atenuação dos dois materiais (água sólida e acrílico). Como o erro encontrado foi em torno de $1 \%$, esse erro foi incluído no controle de qualidade dosimétrico, realizado com a câmara de ionização, permitindo assim que pudéssemos colocar placas de acrílico para compor a espessura total do phantom. Sabe-se que o uso somente de placas de água sólida seria insuficiente para realizar as medidas de forma satisfatória. O phantom foi imageado no equipamento de tomografia computadorizada e as imagens dos cortes foram importadas para a versão 15.6 do software Eclipse da Varian para posterior inserção das seguintes estruturas alvo: Cshape fácil, Cshape difícil, cabeça e pescoço, multialvo e próstata.

Utilizou-se uma câmara de ionização da PTW, modelo TN31010 cujo volume sensível é de $0,125 \mathrm{~cm}^{3}$ para dosimetria absoluta e o EPID (do inglês, Eletronic Portal Imaging Device) para avaliar a distribuição de dose planar. Não foi utilizado filme nas medidas, pois o serviço não possui um densitômetro óptico para a avaliação dos testes.

$\mathrm{O}$ acelerador linear utilizado foi o Halcyon 2.0, de energia máxima de $6 \mathrm{MeV}-\mathrm{FFF}$ da empresa Varian® com duas camadas de Multileaf Colimator (MLC), sendo uma proximal e outra distal com 28 lâminas cada, de largura de $0.5 \mathrm{~cm}$, como mostrado na Figura 2.

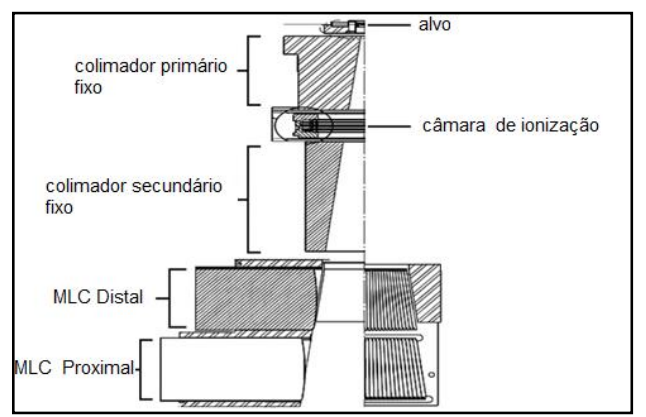

Figura 2. Diagrama esquemático das estruturas de saída do acelerador com ênfase para os MLCs.

Para o planejamento foram utilizadas as imagens DICOM-RT importadas e as estruturas fornecidas pelo protocolo AAPM TG-119 (4),citadas anteriormente para o sistema de planejamento Eclipse. Os planos foram elaborados todos com a única energia disponível $6 \mathrm{MeV}$ de fótons, sem 0 filtro achatador (do inglês FFF- Flattening Filter Free) com dose por fração de 200 cGy, exceto para o planejamento de próstata, para o qual foi utilizada com dose de 180 cGy por fração, aplicando-se o cálculo de heterogeneidade com uma grade de cálculo de $2 \mathrm{~mm}$.

As doses utilizadas para otimização dos planos foram aquelas indicadas no protocolo AAPM TG119(4). No caso das medidas de dose pontual com a câmara de ionização, a comparação entre as doses medidas e planejadas foi realizada em relação à dose prescrita, de acordo com a Equação 1 abaixo, onde $r$ é definido como desvio fracional, que representa a razão da diferença de dose medida com a planejada em relação a dose prescrita.

$$
r=\frac{\left(\text { Dose }_{\text {medida }}-\text { Dose }_{\text {planejada }}\right)}{\text { Dose }_{\text {prescrita }}}
$$

Para a análise das distribuições de doses planares foi utilizado o critério gamma para concordância de $3 \%$ e $3 \mathrm{~mm}$ entre a dose medida via portal e a dose planejada (dose no ponto de referência).

As Figuras de 3 a 11 apresentam as estruturas alvo com seus respectivos planejamentos, com a distribuição de dose para cada uma das estruturas do protocolo TG-119 da AAPM (4).

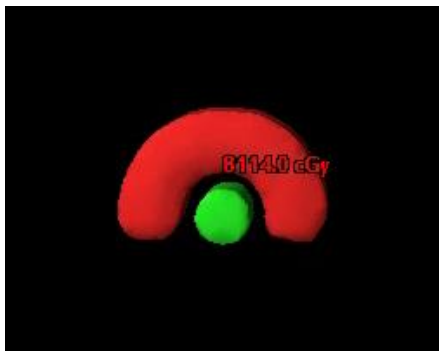

Figura 3. Estruturas para planejamento Cshape (fácil e difícil). 


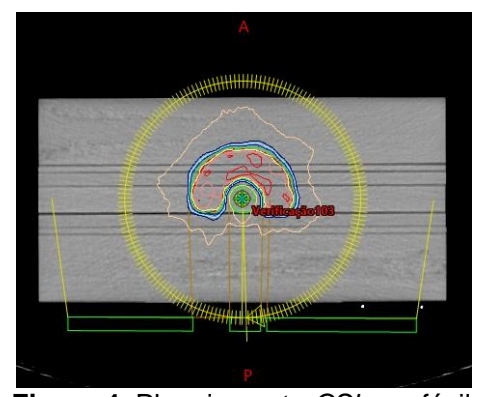

Figura 4. Planejamento CShape fácil

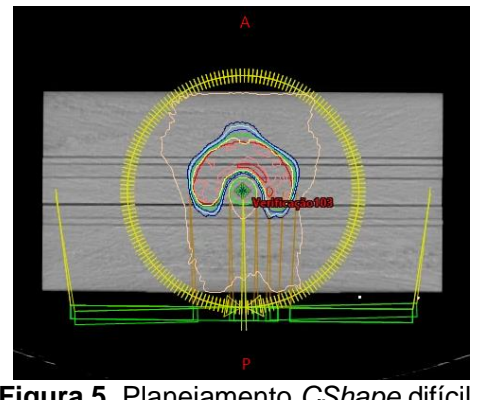

Figura 5. Planejamento CShape difícil.

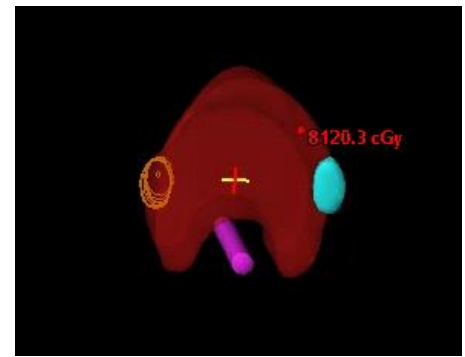

Figura 6. Estruturas para planejamento cabeça e pescoço

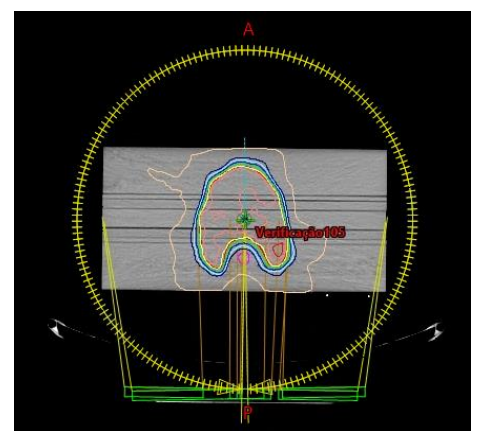

Figura 7. Planejamento cabeça e pescoço.

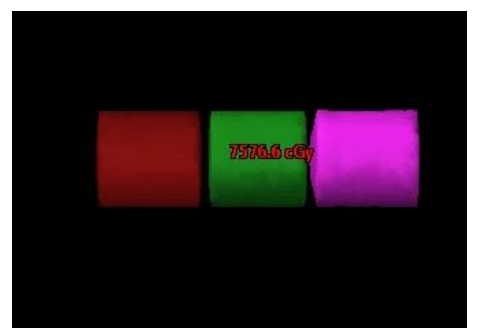

Figura 8. Estruturas para planejamento multialvo

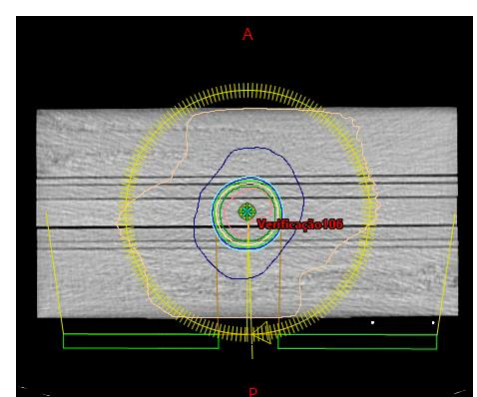

Figura 9. Planejamento multialvo.

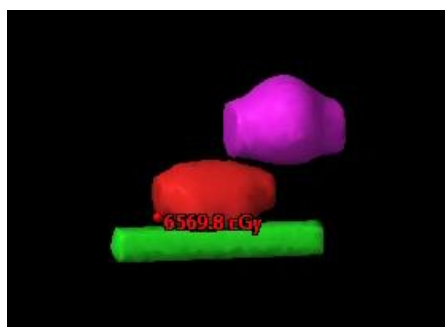

Figura 10. Estruturas para planejamento próstata.

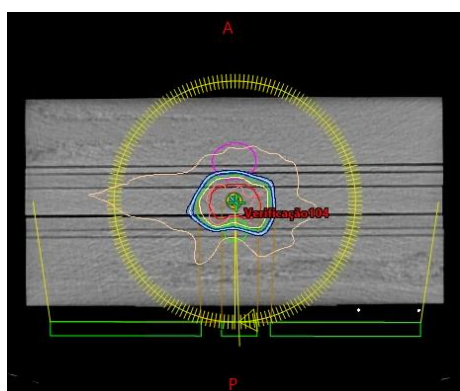

Figura 11. Planejamento próstata

\section{Resultados}

\subsection{Objetivos do Plano}

Para a otimização dos planejamentos de cada teste, foram utilizados os objetivos de dose sugeridos pelo protocolo AAPM TG-119(4).Os valores de referência do protocolo e os valores atingidos neste estudo após a otimização, são apresentados nas Tabelas 1 a 5.

Tabela 1. Planejamento Multialvo

\begin{tabular}{|c|c|c|}
\hline $\begin{array}{l}\text { Parâmetro de } \\
\text { Planejamento }\end{array}$ & $\begin{array}{c}\text { Referência } \\
\text { (cGy) }\end{array}$ & $\begin{array}{c}\text { Atingido } \\
\text { (cGy) }\end{array}$ \\
\hline D99 no alvo central & $\geq 5000$ & 4998 \\
\hline D10 no alvo central & $\leq 5300$ & 5347 \\
\hline D99 no alvo superior & $\geq 2500$ & 2543 \\
\hline D10 no alvo superior & $\leq 3500$ & 3090 \\
\hline D99 no alvo inferior & $\geq 1250$ & 1251 \\
\hline D10 no alvo inferior & $\leq 2500$ & 2316 \\
\hline
\end{tabular}

Tabela 2. Planejamento Próstata

\begin{tabular}{lcc}
\multicolumn{3}{c}{ Tabela 2. Planejamento Próstata } \\
\hline $\begin{array}{l}\text { Parâmetro de } \\
\text { Planejamento }\end{array}$ & $\begin{array}{c}\text { Referência } \\
\text { (cGy) }\end{array}$ & $\begin{array}{c}\text { Atingido } \\
\text { (cGy) }\end{array}$ \\
\hline D95 no PTV & $\geq 7560$ & 7782 \\
D5 no PTV & $\leq 8300$ & 8053 \\
D30 no reto & $\leq 7000$ & 5416 \\
D10 no reto & $\leq 7500$ & 7475 \\
D30 na bexiga & $\leq 7000$ & 3093 \\
D10 na bexiga & $\leq 7500$ & 4955 \\
\hline Fonte: O autor (2021).
\end{tabular}

Tabela 3. Planejamento Cabeca e Pescoco

\begin{tabular}{lcc}
\hline $\begin{array}{l}\text { Parâmetro de } \\
\text { Planejamento }\end{array}$ & $\begin{array}{c}\text { Referência } \\
\text { (cGy) }\end{array}$ & $\begin{array}{c}\text { Atingido } \\
\text { (cGy) }\end{array}$ \\
\hline D90 no PTV & $\geq 5000$ & 5071 \\
D99 no PTV & $\geq 4650$ & 4660 \\
D20 no PTV & $\leq 5500$ & 5365 \\
Dose máxima & $\leq 4000$ & 3667 \\
na medula & & \\
$\begin{array}{l}\text { D50 parótida } \\
\text { direita }\end{array}$ & $\leq 2000$ & 1812 \\
$\begin{array}{l}\text { D50 parótida } \\
\text { esquerda }\end{array}$ & $\leq 2000$ & 1766 \\
\hline \multicolumn{2}{l}{ Fonte: O autor (2021). }
\end{tabular}




\begin{tabular}{lcc}
\multicolumn{3}{c}{ Tabela 4. } \\
$\begin{array}{l}\text { Parâmetro de } \\
\text { Planejamento }\end{array}$ & $\begin{array}{c}\text { Referência } \\
\text { (cGy) }\end{array}$ & $\begin{array}{c}\text { Atingido } \\
\text { (cGy) }\end{array}$ \\
\hline D95 no PTV & $\geq 5000$ & 4998 \\
D10 no PTV & $\leq 5500$ & 5506 \\
D5 na medula & $\leq 2500$ & 2487 \\
\hline Fonte: O autor (2021). \\
\multicolumn{3}{c}{ Tabela 5. Planejamento CShape (difícil) } \\
\hline Parâmetro de & Referência & Atingido \\
Planejamento & (cGy) & (cGy) \\
\hline D95 no PTV & $\geq 5000$ & 5001 \\
D10 no PTV & $\leq 5500$ & 5370 \\
D5 na medula & $\leq 1000$ & 1200 \\
\hline Fonte: O autor (2021).
\end{tabular}

\subsection{Medidas de Dose Pontual}

Os valores medidos de dose pontual com a câmara de ionização semiflex foram avaliados seguindo a Equação 1. A Tabela 6 mostra a comparação entre a dose medida, a dose planejada e a dose prescrita, para cada planejamento, dividida em região de alta e baixa dose.

Tabela 6. Medidas de desvio fracional da dose pontual

\begin{tabular}{lcc}
\hline \multicolumn{1}{c}{ Planejamento } & $\begin{array}{c}\text { Região de Alta } \\
\text { Dose }\end{array}$ & $\begin{array}{c}\text { Região de } \\
\text { Baixa Dose }\end{array}$ \\
\hline Multialvo (centro) & $-0,001$ & - \\
Multialvo (superior) & - & 0,003 \\
Multialvo (inferior) & - & $-0,0131$ \\
Próstata & $-0,002$ & 0,007 \\
Cabeça e Pescoço & $-0,0003$ & 0,019 \\
CShape (fácil) & $-0,006$ & $-0,005$ \\
CShape (difícil) & $-0,016$ & $-0,00026$ \\
\hline \multicolumn{1}{c}{ Fonte: O autor $(2021)$} & &
\end{tabular}

Fonte: O autor (2021).

$\mathrm{Na}$ Tabela 7 são mostradas as médias das medidas de desvio fracional da dose pontual, o respectivo desvio padrão e o limite de confiança, conforme estipulado pelo protocolo AAPM TG119(4).

Tabela 7. Estatística das medidas de desvio fracional da dose pontual e o limite de confiança

\begin{tabular}{|c|c|c|}
\hline & $\begin{array}{c}\text { Região de Alta } \\
\text { Dose }\end{array}$ & $\begin{array}{c}\text { Região de Baixa } \\
\text { Dose }\end{array}$ \\
\hline Média & $-0,003$ & $-0,0014$ \\
\hline Desvio Padrão $(\sigma)$ & 0,0020 & 0,007 \\
\hline $\begin{array}{l}\text { Limite de } \\
\text { confiança }\end{array}$ & 0,006 & 0,015 \\
\hline
\end{tabular}

Fonte: O autor (2021).

O limite de confiança mostrado na Tabela 7 e calculado conforme a Equação 2, é utilizado para descrever o quão próximo o conjunto de medições é concordante com os valores planejados. Para as doses pontuais, a concordância perfeita seria uma razão de diferença de 0,00.

$$
\text { Limite de confiança }=\mid \text { média } \mid+1,96 \sigma
$$

onde $\sigma$ é o desvio padrão da média.

\subsection{Medidas de Dose Planar}

A Figura 12 mostra a distribuição de dose prevista (coluna direita) e medida com o Portal Vision do acelerador (coluna esquerda), para todas as estruturas deste trabalho.

Os valores dos pontos aprovados das distribuições de dose planar medidas encontram-se na Tabela 8.

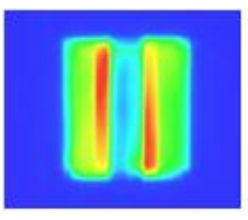

a

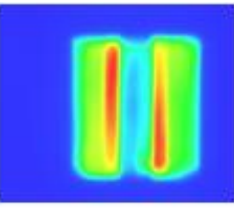

b
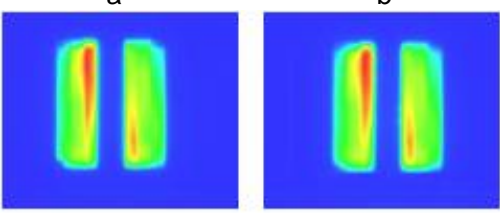

d
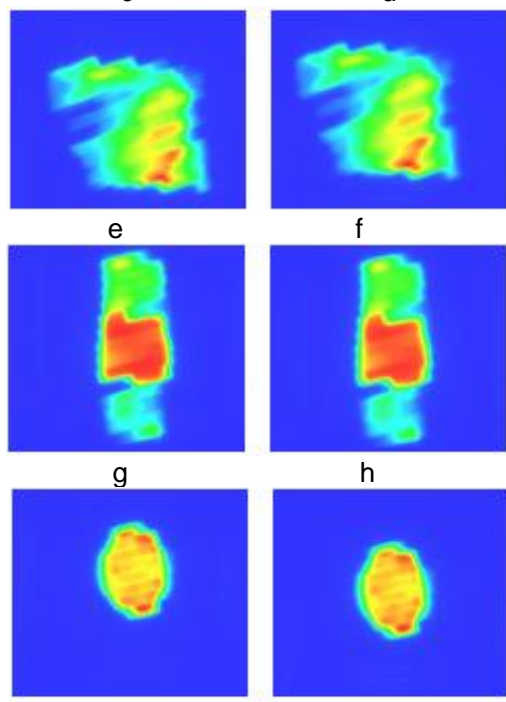

Figura 12 - As figuras da coluna da esquerda são as doses previstas em portal e as da coluna da direita são as doses medidas. (a) e (b) CShape fácil (c) e (d) CShape difícil, (e) e (f) Cabeça e pescoço, (g) e (h) Multi-alvo, (i) e (j) próstata.

Tabela 8. Pontos aprovados pelo critério gamma

\begin{tabular}{lc}
\hline Planejamento & $\begin{array}{c}\text { Percentual de Pontos } \\
(\%)\end{array}$ \\
\hline Multialvo & 98,5 \\
Próstata & 99,3 \\
Cabeça e Pescoço & 96,2 \\
CShape (fácil) & 99,7 \\
CShape (difícil) & 99,9 \\
\hline
\end{tabular}

Na Tabela 9 é mostrada a média, o desvio padrão e o limite de confiança, conforme recomendado pelo protocolo AAPM TG-119(4). Para as análises gamma, a concordância perfeita seria $100 \%$ cujo limite de confiança (CL, do inglês confidence limit) é definido pela Equação 3, que é levemente diferente da equação 2 usada para dose pontual.

Limite de confiança $=(100-$ média $)+1,96 \sigma$

onde média é a porcentagem média de pontos que passam nos critérios gamma e $\sigma$ é o desvio padrão em relação à média. $\mathrm{Na}$ análise gamma, $95 \%$ dos testes devem resultar em aprovação que excedam $(100-C L) \%$, sendo que no presente estudo foi de $96,58 \%$

Tabela 9. Avaliação dos pontos aprovados pelo critério gamma

\begin{tabular}{lc}
\hline & Resultados \\
\hline Média & $98,72 \%$ \\
Desvio padrão $(\sigma)$ & $1,096 \%$ \\
Limite de confiança & $3,42 \%$ \\
\hline Fonte: O autor $(2021)$. &
\end{tabular}




\section{Discussão}

Baseado nos resultados obtidos, pode-se notar que a maior parte dos parâmetros dos planejamentos teve o objetivo alcançado. Segundo a referência do protocolo AAPM TG-119 (4), enquanto outros pontos dos testes de comissionamento não obtiveram sucesso quando comparado à referência. Os pontos de insucesso foram: D99 no alvo central, D10 no alvo central e D99 no alvo inferior para o caso da estrutura Multi-Alvo e D95 no PTV e D10 no PTV para a estrutura CShape (fácil). Apesar do insucesso de alguns pontos dos testes, ao observarmos o valor obtido comparado ao valor de referência, ele não chega a $1 \%$ de diferença, de forma que não é possível invalidar tal teste de comissionamento.

Com respeito às medidas de dose pontual, primeiramente podemos citar o fator de correção $K t p$, cuja expressão é dada pela Equação 4, que é um número adimensional que leva em consideração os fatores temperatura e pressão do ambiente no momento da medida.

$$
K t p=\left(\frac{(273,2+T)}{273,2+22}\right) \cdot\left(\frac{(1013,25)}{P}\right)
$$

onde $\mathrm{T}$ é a temperatura medida em ${ }^{\circ} \mathrm{C}$ e $\mathrm{P}$ é a pressão medida em $\mathrm{hPa}$

As medidas de dose pontual foram realizadas com pressão de $1020 \mathrm{hPa}$ a uma temperatura de 22,4 ${ }^{\circ} \mathrm{C}$, pois variações muito bruscas na pressão e temperatura poderiam afetar as cargas coletadas provocando medidas de dose inconsistentes. No entanto, cabe ressaltar que não há na literatura estudos que mostram intervalos de valores mínimos e máximos de pressão e temperatura para que as medidas de dose sejam consistentes.

As medidas de dose pontual com câmara de ionização mostraram uma variação de $0,1 \%$ até $0,6 \%$ para alta dose e 0,2 até $1 \%$ para baixa dose. A média para região de alta dose foi de $0,3 \%$ e para região de baixa dose foi de $0,1 \%$. Esses baixos valores percentuais mostram que mesmo que haja diferenças entre as doses medidas em relação as doses planejadas são diferenças mínimas; em um caso se chegou a $1 \%$ de diferença, mostrando que 0 planejado está de acordo com o medido. Se analisarmos a outra abordagem de validação, que é pela análise gamma, temos um intervalo entre 96,2 $\%$ e $99,9 \%$ do percentual de pontos aprovados pela análise, de forma que esses resultados obtidos para os casos propostos pelo protocolo AAPM TG-119(4), evidenciam o sucesso dos testes.

O conjunto de casos propostos pelo TG-119 é muito útil para se entender e comparar diferentes módulos de otimização, permitindo, assim, a possibilidade de explorar os vários níveis de complexidade dos planejamentos nos casos, mostrando a capacidade de otimização. As metas do TG-119 ajudam a estabelecer uma linha de base e dados de comissionamento tomados como referência para IMRT. Eles também são úteis para se obter confiança em novas modalidades como VMAT para que se possa testar seus recursos no estágio de implementação pré-clínica. Os programas de VMAT, como são feitos no TG-119, exigiriam estudos multi-institucionais e de vários fabricantes também (5). A implementação dos testes de comissionamento no Halcyon não apresentou nenhuma dificuldade, sendo a execução mais rápida em relação aos outros aceleradores lineares em virtude da velocidade do sistema de lâminas, alta taxa de dose e do giro do gantry.

\section{Conclusões}

Os objetivos dos planos sugeridos pelo protocolo AAPM TG-119 foram alcançados de forma satisfatória para o acelerador linear Halcyon 2.0 instalado no serviço de radioterapia, apesar de alguns pontos não terem sido alcançados, possivelmente por questões dos algoritmos de cálculo do software ao final da otimização.

Sobre as medidas de dose pontual e dose planar, para avaliação da reprodução dos planos realizados pelo sistema, foram plenamente satisfatórias, com média de 0,003 para região de alta dose e 0,0014 para região de baixa dose, ficando dentro da tolerância do protocolo TG-119 da AAPM, cujo valor é 0 .

Em relação às medidas de dose planar, o percentual de aprovação para todos os planejamentos sugeridos pelo protocolo TG-119 da AAPM está dentro dos limites de tolerância estipulados pelo protocolo que estipulava um mínimo de $95 \%$ para aprovação.

Por meio desses valores, tanto para dose pontual quanto para dose planar pode-se afirmar que o sistema utilizado para modelagem do planejamento é adequado e satisfatório, sendo o plano reprodutível no acelerador linear fazendo com que o objetivo deste trabalho fosse alcançado, validando, portanto a entrega de planos gerados em um sistema Halcyon 2.0 com 6FFF.

\section{Agradecimentos}

Agradeço à equipe do serviço de radioterapia do Hospital São Lucas da PUCRS, especialmente a equipe de física médica que contribuiu para elaboração deste trabalho.

\section{Referências}

1. Watanabe, Érika Yumi.Com paração da técnica de radioterapia em arco modulada volumetricamente (VMAT) em relação à técnica de radioterapia de intensidade modulada (IMRT) para tumores de próstata e cabeça e pescoço.Tese de Doutorado.São Paulo. Universidade de São Paulo.2015

2. Otto K. Volumetric modulated arc therapy: IMRT in a single gantry arc. MedPhys. 2008 Jan;35(1):310-7.

3. Zeppelini C, Furnari L. Comissionamento de IMRT - Aplicação do TG-119 da AAPM. Revista Brasileira de Física Médica. 2013; 7(2):57-60

4. AAPM - American Association of Physicists in Medicine. TG119 IMRT Comissioning Tests: Instructions for Planning, Measurement, and Analysis. 2009.

5. Mynampati,D.K., Yaparpalvi,R., Hong,L., Kuo,H.C. Mah.D. Application of TG 119 to volumetric arc therapy (VMAT). Journal of Applied Clinical Medical Physics, 2012 13(5).:108-16

\section{Contato:}

Diego Santos Teixeira

Hospital São Lucas da PUCRS - Serviço de Radioterapia

E-mail: Diego.Teixeira86@edu.pucrs.br 